\title{
Expresión del futuro en el español oral de Chile actual: aproximación cognitivista a un caso de gramaticalización ${ }^{*}$
}

\author{
Matías Jaque $^{* *}$
}

\begin{abstract}
Resumen
En el presente trabajo se aborda el proceso de gramaticalización de los exponentes de futuro en español de Chile, considerando un repertorio de formas sintéticas y analíticas: -ré, ir $a+$ inf, tener que + inf, querer + inf, y haber que + inf. Mediante el análisis de dos corpus orales del español de Chile, de 1970 y 2009, concluimos que la forma ya gramaticalizada en -rése encuentra en retroceso como exponente de temporalidad, frente a la alternativa analítica ir a + inf. En tanto, el resto de las formas perifrásticas sostiene una competencia discursiva que puede conducir a nuevos procesos de gramaticalización. Para dar cuenta de ello, se propone una distinción cognitiva entre dos formas de conceptualizar la temporalidad: el tiempo fenomenológico, que correspondería a la experiencia directa del tiempo; y el tiempo simbólico, derivación más abstracta que permite ordenar intervalos respecto de un centro deíctico.
\end{abstract}

Palabras clave: tiempo futuro, aspecto y modalidad, perífrasis, gramaticalización, cognición temporal.

\section{The expression of future in present-day oral Spanish: a cognitivist approach to a case of grammaticalization}

\begin{abstract}
This work addresses the grammaticalization process of future exponents in Chilean Spanish. In particular, we study the synthetic option in -ré and the analytic in ir a + inf, in comparison with other periphrastic options: tener que + inf, querer + inf y haber que + inf. By analyzing two oral corpora from Chilean Spanish (from 1970 and 2009), we conclude that the grammaticalized form in -ré is disappearing as a future exponent. In contrast, the form in ir a + inf has advanced in the grammaticalization process as the main expression of future, while the alternative periphrastic forms exhibit a competence in discourse that could lead to new grammaticalization processes. We account for this process by distinguishing two ways of temporal conceptualization: phenomenological time, which corresponds to direct experience of time, and symbolic time, an abstract derivation which allows interval ordering with respect to a deictic center.
\end{abstract}

Keywords: future tense, aspect, modality, grammaticalization, temporal cognition.

Recibido: 10-10-2016 Aceptado: 12-12-2016

\footnotetext{
* La elaboración de este trabajo se vio parcialmente beneficiada del proyecto "Relaciones entre aspecto y modalidad en español: contrastes tipológicos y bases cognitivas" (U-I-24/15). Agradezco las observaciones de Guillermo Soto y Carlos Zenteno, que leyeron una versión previa como integrantes del tribunal de tesis de magíster que lo evaluó (Universidad de Chile, 2012), y en el que en buena medida se basa el presente artículo. Quisiera dedicar este trabajo a la memoria de Carlos Zenteno, profesor de lengua inglesa de la Universidad de Chile. Las deficiencias y errores existentes corresponden exclusivamente al autor. ** Chileno. Doctor en Filosofía y Lingüística. Universidad Autónoma de Madrid. Académico asistente Universidad de Chile. matjaque@uchile.cl
} 


\section{Introducción}

El vínculo diacrónico existente entre las categorías verbales de aspecto, modalidad y tiempo ha sido extensamente abordado (Heine, Claudi y Hünnemeyer, 1991; Heine, 1993; Bybee et al., 1994, entre otros). En el caso del futuro, ciertos exponentes de modalidad (en especial la orientada al agente, sea deóntica o volitiva) y aspecto (inminencial) suelen evolucionar hacia la expresión de una relación de posterioridad entre el punto de referencia y el momento de habla. Tales patrones poseen interés no solo por lo que revelan acerca de los mecanismos regulares de cambio lingüístico, sino por lo que estos pueden enseñar, de modo general, sobre la relación entre cognición y lenguaje. De este modo, existe una relación no solo entre las categorías lingüísticas de modalidad, aspecto y tiempo, sino entre tales categorías y los ámbitos cognitivos que les subyacen (Heine, 1993). Las regularidades diacrónicas pueden revelarse útiles, así, como indicios de regularidades en los procesos de conceptualización de la realidad.

En el presente estudio seguimos esta agenda, enfocándonos en el caso del proceso de gramaticalización asociado a la categoría de futuro. Siguiendo la indicación de Fleischmann (1982), analizamos el funcionamiento de esta categoría desde un punto de vista cognitivo que puede hallar expresión de distintos modos, sean sintético-flexivos (-ré) o analítico-perifrásticos (voy a cantar), y pertenezcan o no a lo que, bajo criterios formales, puede considerarse parte del sistema de expresión temporal de la lengua española. Así, junto a estas formas canónicas de expresar futuro, nos interesa ponderar cómo el grado avanzado de gramaticalización de una unidad puede verse acompañado por procesos de gramaticalización incipiente en otras perífrasis que, tomando en cuenta la documentación histórica del español y otras lenguas, son igualmente aptas para servir de expresión a esta categoría temporal. Es el caso de querer + inf, tener que + inf, a las que se suma, por motivos que se expondrán más abajo, haber $q u e+$ inf. En los apartados siguientes, defenderemos la idea de que la evolución de los exponentes de futuro opera como un conjunto armónico, en el que el mayor grado de gramaticalización de un exponente puede favorecer la puesta en marcha o la agudización de un proceso análogo en otros. Aunque no contamos con datos para confirmar categóricamente esta afirmación, proponemos una estrategia para examinar el progreso paralelo de distintas expresiones, según expondremos en el apartado §2. 


\section{Consideraciones teóricas}

\subsection{La gramaticalización del futuro: inferencia y metáfora}

En los estudios dedicados a la gramaticalización del futuro pueden distinguirse, al menos, dos estrategias explicativas. Por un lado, algunos autores enfatizan el papel que desempeñan las inferencias discursivas en la fijación de nuevos contenidos gramaticales; por otro, se ha destacado el papel de las metáforas conceptuales en la selección de la fuente léxica (comúnmente, verbos de movimiento). Suscribiremos aquí una tercera opción, inspirada en el trabajo de Fleischman (1982) y, más recientemente, Evans (2005), de acuerdo con la cual la expresión del tiempo viene determinada por restricciones cognitivas elementales que poseen prioridad tanto sobre las metáforas como sobre los mecanismos inferenciales que tienen lugar en el discurso.

En la aproximación de Bybee et al. (1994) y Bybee $(1998,2003)$, se concede especial importancia al papel que juega el incremento de la frecuencia de uso en la emergencia de significados gramaticales. En particular, la frecuencia contribuye a la automatización de las inferencias pragmáticas que se dan en el discurso. Por ejemplo, en la gramaticalización de be going to como operador de futuro en inglés se produce con recurrencia la inferencia de que el movimiento a un destino lleva consigo una intención y que, en última instancia, aquello que se intenta puede producirse en el futuro (cf. Cuenca y Hilferty, 1999). La automatización de esta inferencia, favorecida por la frecuencia de uso, contribuye a que ese contenido deje de ser un valor facultativo añadido y pase a considerarse como parte integrante del significado de la construcción, con el consecuente reanálisis de la estructura $[t o \mathrm{~V}]$, que pasa de cláusula final a núcleo del predicado verbal. El hecho de que los seres humanos realicemos, en general, inferencias similares en situaciones análogas, explicaría el establecimiento de rutas de gramaticalización relativamente estables, tales como la que, para el futuro, se sintetiza a continuación (basado en Bybee $(1998,2003)$, Bybee y Pagliuca (1987) y Bybee et al. (1991)):

Figura 1.

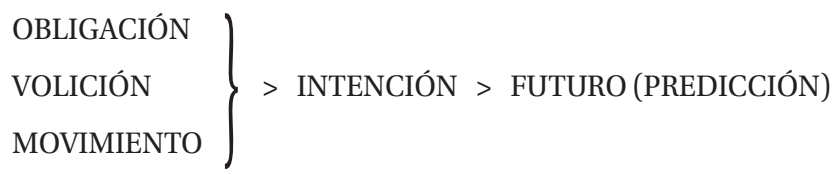


Una segunda aproximación enfatiza, como decíamos, el papel de las metáforas conceptuales (Lakoff y Johnson, 1980; Grady, 1997; Heine, 1993) en la gramaticalización de verbos de movimiento que expresan futuro. Un ejemplo para el español es el trabajo de Melis Flores y Bogard (2003) y Melis (2006) ${ }^{1}$. De acuerdo con los autores, en el proceso de gramaticalización de la forma ir $a+$ inf, que se remonta a los orígenes del español, no es necesario postular un proceso inferencial que tome como fuente una construcción locativa que exprese un destino, en contraste con la propuesta de Bybee et al. (1994). Para Melis (2006), si este fuera el caso, se esperaría un incremento de la construcción locativa en las etapas iniciales, seguido de su decaimiento conforme la inferencia discursiva permitiera un reanálisis sintáctico. No obstante, en su estudio diacrónico de corpus, se encontró que la incidencia de la construcción locativa es relativamente estable a lo largo de los siglos, desde un $42 \%$ en el $\mathrm{Cid}$, hasta un $38 \%$ en textos del siglo XX. Consiguientemente, Melis (2006) concluye que la teoría correcta corresponde a un proceso de metaforización que se aplica directamente sobre la fuente léxica ir ya desde los orígenes, sin pasar por una etapa intermedia de inferencias a partir de una construcción locativa en que el destino favorezca una lectura de intención.

Las dos propuestas arriba sintetizadas tienen obvias limitaciones, al menos si se pretende acogerlas como modelos explicativos generales. De un lado, el énfasis en la frecuencia y la fijación de inferencias deja sin atender las motivaciones cognitivas o funcionales que favorecen, justamente, la aparición de ciertas inferencias². Así, aun cuando exista cierta esperanza de formular un procedimiento mecánico una vez que la frecuencia de una construcción se dispara, es difícil explicar la universalidad de ciertas rutas de gramaticalización si no existe una base común que promueva tales procesos inferenciales. Por otra parte, dicha moti-

\footnotetext{
1 La propuesta general de los autores queda expresada en la siguiente cita: "Las metáforas que los hablantes de todas las épocas generan combinando el verbo de movimiento ir con una meta figurativa, sea en forma nominal o infinitiva, ilustran la tendencia universal de los seres humanos a conceptualizar los cambios que se desarrollan en el tiempo en términos de movimientos efectuados en el espacio" (Melis et al., 2003, p.28).

2 Al respecto, Bybee et al. (1994) incluyen observaciones generales sobre las motivaciones funcionales que guían la puesta en marcha de ciertas inferencias, aunque estas no se desarrollan en profundidad. Considérese, por ejemplo, la cita siguiente, referida al cambio del perfecto (anterior) en pretérito:

Such changes occur because of the way language is used. The anterior conveys the sense of past or perfective but includes a special flavor of relevance or proximity to the present or current situation. Thus if a speaker wishes to frame his or her contribution AS THOUGH it were highly relevant to current concerns, then the speaker might use the anterior more often than would be strictly necessary for the communication of the propositional content of the message. (p.86-87).
} 
vación básica tampoco puede provenir exclusivamente de la teoría de la metáfora, toda vez que, como se observa en la figura 1, el movimiento es solo una de las fuentes léxicas que, de modo recurrente, emplean las lenguas para producir expresiones gramaticales de futuro. Respecto de las nociones de volición y obligación, ¿debemos extender la noción de metáfora conceptual? ¿O solo conseguiríamos con ello debilitar el alcance explicativo que su empleo tiene en el caso de los verbos de movimiento?

\subsection{Restricciones cognitivas básicas}

De acuerdo con Evans (2005), la fenomenología del tiempo corresponde a una experiencia básica que precede a su conceptualización metafórica a través de una fuente léxica concreta. Esta fenomenología tendría correlatos neurofisiológicos claros en la emergencia de ventanas de sincronización neuronal de unos pocos milisegundos, correspondientes a lo que intuitivamente consideramos el presente (Varela, Thompson y Rosch, 1991; Varela, 1995; Pöppel, 1994). De este modo, para Evans (2005), la teoría de la metáfora falla en conceder a la percepción de objetos externos un estatus cognitivo más elemental que la propia percepción del tiempo: "[w] must question the view that the concept of time is derived from event perception and that cognitively temporal experience is less basic than sense-perceptory experience" (2005, p.23).

Esta idea es abordada igualmente por Varela (1995), quien, basándose en aportes de Husserl (1905), expone un modelo que busca dar cuenta del carácter primigenio y no derivado del tiempo. Aquí, el presente tiene preeminencia en nuestra fenomenología temporal básica, y de él se deriva o proyecta el resto de los tiempos. De acuerdo con Husserl (1905), la extensión del presente hacia el pasado se denomina retención; la extensión del presente hacia el futuro, protención ${ }^{3}$. Ambos integran lo que Varela (1995) llama presente espeso, y deben distinguirse, para Husserl (1995),

\footnotetext{
3 Por motivos de espacio, no podemos hacer justicia a la sutileza del análisis fenomenológico de Husserl (1905) para las categorías de retención y protención. Baste mencionar que el problema al que se enfrenta el filósofo es dar cuenta de la percepción de "objetos temporales" (el movimiento de un objeto, una sinfonía, etc.). De acuerdo con Husserl (1905), la percepción unificada del movimiento de un objeto no puede coincidir ni con la permanencia literal de los momentos anteriores (en cuyo caso observaríamos un objeto continuo a lo largo del espacio) ni con la percepción discontinua de diversos presentes (en cuyo caso percibiríamos un objeto reapareciendo en diferentes puntos del espacio, en stop motion). La idea, por lo tanto, es que el pasado inmediato sigue impactando en el presente, del que se desprende de modo gradual. Según Varela (1995), la retención correspondería a la memoria ecoica distinguida por la psicología cognitiva. Para más discusión, véase Rodemeyer (2003), Zahavi (2003), entre otros.
} 
tanto del acto de rememoración, que consiste en la re-presentación de un momento genuinamente pasado; como del acto de expectativa, que correspondería a un evento todavía no acaecido. Estos conceptos pueden esquematizarse como sigue:

Figura 2

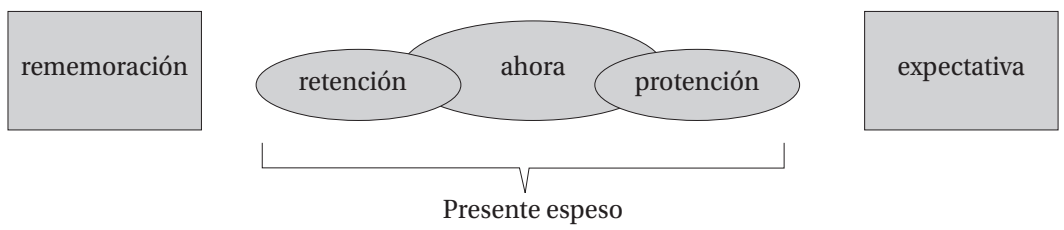

La proyección del ahora en retención y protención constituye la forma misma del presente y posee un carácter básico e involuntario; la retención es, así, un "constante escorzamiento" (p. 68) del ahora que se transforma en pasado. En cambio, no cabe hablar de un paso constante e involuntario de la percepción del ahora al acto de fantasía constituido por la rememoración (íbid). El presente espeso corresponde, así, a la percepción fundamental del paso del tiempo, mientras que los actos de fantasía son derivados y facultativos.

Basándonos en estas ideas, proponemos una oposición conceptual básica de tipo cognitivo que, según defendemos, tiene un correlato en la expresión lingüística del tiempo. En primer lugar, distinguiremos entre tiempo fenomenológico y tiempo simbólico. El primero corresponde, grosso modo, al concepto de presente espeso desarrollado por Varela (1995). Se trata, así, de la experiencia directa del tiempo, en la que el presente posee preeminencia sobre el pasado y el futuro, tiempos que pueden entenderse como proyecciones de aquel. Por su parte, el tiempo simbólico corresponde a una derivación cognitiva posterior, en la que ordenamos intervalos y eventos con independencia de la experiencia directa del presente (y se aproxima, por tanto, a los actos de rememoración o expectativa de Husserl (1905)).

Desde un punto de vista fenomenológico, existe una radical asimetría entre pasado y futuro: el segundo corresponde a un dominio ontológico potencial o no realizado hacia el cual solo podemos tener, en principio, una disposición afectiva (Rodemeyer, 2003). En términos simbólicos, en cambio, la única diferencia entre pasado y futuro radica en la posición deíctica que ocupan respecto del presente. Desde un punto de vista lingüístico, podemos interpretar los tiempos verbales plenamente gramati- 
calizados (cantaré o cantó) como exponentes de tiempo simbólico, en los que la referencia deíctica a intervalos anteriores o posteriores al presente es inequívoca (cf. Klein, 1994). Sin embargo, en la medida en que la mención directa del futuro transgrede la experiencia inmediata del tiempo, su referencia requerirá de la compensación efectuada por exponentes que lo vinculen al dominio del tiempo fenomenológico. Estos exponentes corresponderán, típicamente, a formas perifrásticas cuyo auxiliar está conjugado en presente, y que expresan contenidos ya sea modales (actitudes o deseos del hablante hacia el contenido proposicional, v.gr. lat. cantare habeo, esp. tengo que cantar), ya sea aspectuales (en especial, de tipo inminencial, que vincula el evento posterior con las características objetivas del presente; v.gr. cuidado, que se va a caer). En ambos casos, la operación cognitiva subyacente consistiría en el redireccionamiento del tiempo simbólico al tiempo fenomenológico, cuestión que se verifica con particular fuerza en la inestabilidad (formal y semántica) del paradigma de futuro en diversas lenguas (cf. Cartagena, 1995-1996; Escandell, 2010).

A ello apunta, si bien desde un marco conceptual diferente, Fleischman (1982) cuando sugiere que:

[a]n apposite metaphor for viewing the relationship, or balance, between temporality and modality in futures is the mathematical notion of a whole, where the parts may vary in inverse relation to each other, but the sum of the parts must remain constant. [...] However, the more temporalized a given form becomes, the weaker its modal force, and vice versa. If the balance shifts sufficiently in the direction of temporality, speakers may eventually be motivated to seek out new forms -frequently periphrases combining a modal auxiliary and a full verb- through which to restore the modality that has been lost in the process of temporalization [...] (p. 31).

Así, la ruta de gramaticalización del futuro se iniciaría con un exponente de modalidad (siguiendo a Fleischman (1982) o aspecto (cf. Melis et al., 2003), reflejo de una restricción cognitiva básica e independiente, en principio, tanto de los mecanismos pragmático-discursivos que permiten fijar nuevos significados como del proceso de metaforización que pueda aplicarse a algunas de las fuentes léxicas que conceptualizan el tiempo (v.gr. movimiento). El tipo de modalidad pertinente será aquel que permita expresar una conexión entre el presente fenoménico y el futuro; típicamente, la obligación o el deseo (modalidad interna u orientada al 
agente). Nótese, pues, que este origen diacrónico no es incompatible con la idea de que el futuro, en una etapa posterior de gramaticalización, puede dar lugar a valores modales de tipo epistémico (modalidad externa u orientada al hablante). Dicha ruta se sintetiza en la siguiente figura:

Figura 3

$$
\left\{\begin{array}{l}
\mathrm{M} \\
\mathrm{A}
\end{array}\right\} \quad>\mathrm{T}_{\text {fut }}>\mathrm{M}
$$

Según veremos, la forma en -ré parece haber abarcado un ciclo completo, al pasar de una fuente modal deóntica (cantare habeo) a un exponente de tiempo simbólico futuro (cantaré) a, finalmente, un exponente de modalidad epistémica (estará en su casa, ¿no?).

Es importante destacar que la propuesta teórica aquí sintetizada no constituye una alternativa absoluta ni a la teoría centrada en la generación de inferencias ni a la de la metáfora conceptual. Más bien, prioriza el impacto de la fenomenología temporal en el proceso de gramaticalización, asignando a los factores restantes un nivel explicativo distinto. Así, proponemos que el principal factor que determina los reajustes en el paradigma verbal de futuro responde a una necesidad de compensación entre ambas formas de cognición temporal: simbólica, en que se prioriza la localización de eventos e intervalos; y fenomenológica, en que cobra realce la experiencia del presente. Cuanto más se orienta una forma hacia el polo del tiempo simbólico, más se agudiza el proceso de gramaticalización de otras formas que, en principio, sean sensibles a la fenomenología temporal enraizada en el presente. En este sentido, la metáfora tiempo-espacio corresponde a un mecanismo a través del cual puede (pero no debe) expresarse esta restricción cognitiva básica, pero, como parece ser el caso, no agota las fuentes conceptuales a través de las que se expresan nuevas formas de futuro. En un nivel explicativo más superficial, las unidades léxicas en las que se expresa el futuro adquieren nuevos valores temporales mediante la fijación de inferencias que tiene lugar en la interacción discursiva. El modelo propuesto puede sintetizarse, en el siguiente esquema: 
Figura 4

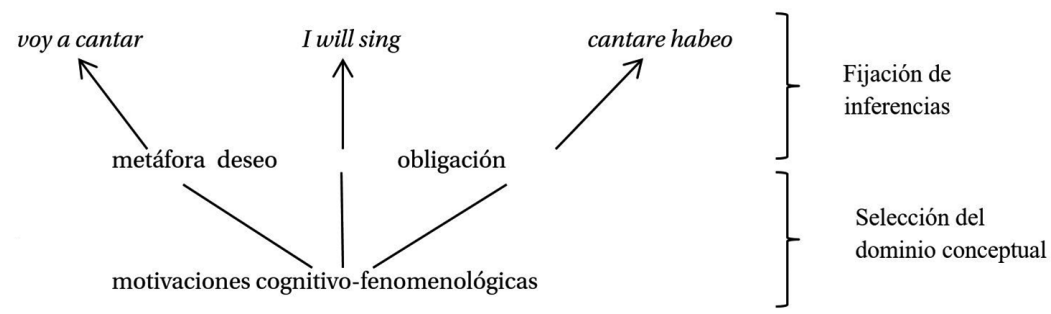

Si el proceso de gramaticalización de los exponentes de futuro resulta de un proceso más básico de compensación entre el tiempo fenomenológico y el tiempo simbólico, es de esperar que las distintas construcciones no sufran procesos de gramaticalización de forma completamente aislada (por un aumento azaroso de la frecuencia o por la recurrencia de ciertas inferencias), sino que se observe un proceso de conjunto más armónico en el que la culminación de un proceso sea el puntapié inicial para el inicio de otro. Esta predicción distancia nuestra propuesta de las anteriores que hemos revisado ${ }^{4}$. En los apartados siguientes revisaremos una estrategia concreta con la que estas cuestiones pueden ser abordadas.

\section{Formas con referencia de futuro en el español actual de Chile}

Pasemos a presentar el análisis de las formas con referencia de futuro en el español actual de Chile. Como ha sido adelantado, hemos realizado una descripción de dos corpus orales separados por 39 años, que nos permitirán evaluar el mecanismo de gramaticalización de estos exponentes a la luz de las consideraciones teóricas expuestas más arriba. En particular, propondremos una estrategia de análisis que permita obser-

4 Cabe mencionar que la perífrasis ir a + inf se empleó esporádicamente durante siglos, hasta conocer un aumento explosivo de su frecuencia durante el siglo XIX. Considérese que, todavía en el siglo XVII, Correas (en Fernández de Castro, 1999 n. 9) observaba que

El futuro de indicativo siempre es conpuesto en una dizion del infinitivo de cada verbo, $\mathrm{i}$ del presente del verbo aver, é, as, á, emos, eis, an, como en amaré, amarás, temeré, consumiré, \&c. [...]. Suelese deshazer la conposizion anteponiendo el presente é, as, al infinitivo entremetida la preposición de, é de amar, as de temer, á de consumir [...] (p.72).

Este testimonio hace pensar que el carácter modal (deóntico) del futuro en -ré pertenecía todavía al sentimiento lingüístico sincrónico de la época, presumiblemente perdido ya por completo durante las décadas posteriores. Así, el auge de la frecuencia de ir $a+$ inf no sería independiente de la suerte de otros miembros del sistema de referencia de futuro. Un fenómeno similar intentaremos detectar para el español contemporáneo. 
var cómo, en el proceso de cambio lingüístico, las formas que expresan futuro operan como un conjunto armónico en el que las construcciones más gramaticalizadas motivan la puesta en marcha de mecanismos gramaticalizadores en otras.

\subsection{Delimitación del significado temporal de las formas}

Al hablar de formas con referencia al futuro, empleamos aquí el término "referencia" de manera suficientemente vaga como para agrupar expresiones con un valor claramente temporal junto a otras que combinan inferencias de tipo temporal con valores semánticos de carácter modal o aspectual. Para distinguir entre ambas opciones, nos basaremos en un sencillo criterio semántico empleado por Comrie (1985), según el cual los valores temporales inferidos son cancelables, no así aquellos que forman parte del significado de una expresión. En consecuencia, solo en el primer caso será admisible el empleo de una cláusula introducida por la conjunción adversativa pero que niegue la ocurrencia del evento futuro aludido. Consideremos los ejemplos siguientes:

1. a. \#Mañana cantaré en el bar, pero no lo haré.

b. \#Mañana voy a cantar en el bar, pero no lo haré.

2. a. Mañana tengo que cantar en el bar, pero no lo haré.

b. Mañana quiero cantar en el bar, pero no lo haré.

c. Mañana hay que cantar en el bar, pero no lo haré.

En las expresiones de (1), la introducción de las cláusulas con pero conduce a enunciados semánticamente contradictorios. Esto indica que, en el caso de la perífrasis ir $a+$ inf, la noción de futuro ha pasado a ser parte del significado de la forma, por lo cual podemos considerarla un exponente de tiempo simbólico, como sucede con la variante sintética en -ré. En cambio, en las tres formas de (2), la cancelación del valor de futuro es plenamente aceptable, indicando que se trata de una inferencia asociada a un valor prioritario de tipo modal (deóntico en el caso de tener $q u e+$ inf y hay que + inf; volitivo en el de querer + inf $)^{5}$.

5 El repertorio de formas perifrásticas que hemos considerado en nuestro estudio no agota aquellas formas que podrían tener un valor secundario de futuro. Entre otras, podemos mencionar deber + inf, haber de + inf y pensar + inf. No las hemos considerado, por tener una frecuencia bastante baja en ambos corpus, cuestión que no nos permitía alcanzar ninguna generalización descriptiva. Un caso aparte corresponde al uso del presente de indicativo, que sí corresponde a una forma altamente productiva de aludir al futuro y que, a diferencia de otras perífrasis, no permite cancelar este significado (v.gr. \#Mañana canto en el bar, pero no lo haré). Para una consideración del presente en el conjunto de formas con referencia de futuro, véase Orozco (2007). 
Los contrastes de (1) y (2) permiten concluir que el valor inminencial de la perífrasis ir $a+$ inf, vigente, siguiendo a Melis (2003), hasta fines del siglo XIX, ha dejado de ser el principal. De todos modos, cabe mencionar que los valores anteriores de una forma gramaticalizada no desaparecen del todo (cf. Bybeeet al., 1994). De este modo, existen algunos contextos en los que, desde el punto de vista semántico, la forma analítica no es sustituible por la forma sintética:

3. a. ¡Apúrate, que $\{$ van a ser/\#serán $\}$ las cinco!

¡Cuidado, te $\{$ vas a caer/\#caerás\}!

En estos casos, donde el hablante pretende comunicar inminencia de un estado de cosas futuro, la opción sintética se percibe como altamente marcada. Este contraste no parece depender del grado de (in)formalidad empleado por el hablante, sino efectivamente de un contraste aspectual.

También es interesante mencionar que, en ciertos contextos, existen restricciones gramaticales que bloquean la forma sintética, pero no la forma analítica. En tales casos, la perífrasis conserva un valor únicamente inminencial. Así sucede con las cláusulas adverbiales introducidas por cuando, que rechazan el modo indicativo y, por tanto, el futuro en -ré:

(4) Cuando $\left\{{ }^{*}\right.$ te casarás/te vayas a casar\}, te presto mi terno blanco.

Como se aprecia en (4), el valor de la forma perifrástica es exclusivamente aspectual: el hablante no sitúa el evento en un momento futuro indeterminado, sino que denota el momento inmediatamente anterior a su realización.

Al margen de estos contextos, en que el valor aspectual originario de la perífrasis parece conservarse, los datos de (1) indican que ir $a+\inf$ se ha gramaticalizado como un exponente de tiempo futuro. Somos conscientes de que, al adoptar esta conclusión, nos distanciamos de otros estudios que proponen algún tipo de oposición semántico/funcional en el que la forma perifrástica, frente a la forma en -ré, codifica mayor certidumbre, cercanía temporal o asertividad (cf. Sedano, 1994; Fernández de Castro, 1999; Bentivoglio y Sedano, 2015). De acuerdo con el estudio de Lastra y Martín-Butragueño (2010), realizado en Ciudad de México, si bien existen variables lingüísticas que parecen favorecer la selección de la forma perifrástica (cercanía temporal, seguridad), el empleo de esta es predominante entre hablantes hombres jóvenes con bajo nivel educacional, resultados que los autores interpretan como un cambio desde abajo en sus fases finales (cf. Bentivoglio y Sedano, 2015, p.175). Esta 
conclusión es concordante con otros estudios, como el de Melis (2006), que atribuyen un mayor peso de factores funcionales a etapas previas del proceso de gramaticalización (siglo XIX y principios del XX); en la actualidad, presumiblemente nos encontremos en una etapa en que la forma perifrástica corresponda a la opción no marcada de referencia al futuro (cf. Orozco, 2007).

En el caso del español de Chile, en un estudio previo realizado en textos de prensa (Jaque, 2012), pudimos observar que la variable que con mayor solidez permite predecir la selección de la forma perifrástica es el carácter oral (recogido en discurso directo) frente al escrito (es decir, correspondiente al cuerpo de la noticia). En particular, el discurso directo concentraba 36 ocurrencias de la opción perifrástica, frente a 7 de la opción sintética; a la inversa, el cuerpo de la notica recogía solo 5 casos de futuro perifrástico, frente a 73 de la forma sintética. Este patrón apunta a una fuerte distribución diafásica, más que semántico-funcional, de ambas opciones. En el presente estudio, según veremos más adelante, la opción abrumadoramente predominante en la oralidad es la perifrástica, con independencia de que, en algunas de sus ocurrencias, se preserven valores aspectuales o modales como los apuntados más arriba.

\subsection{Indicadores de gramaticalización}

Junto a la delimitación del valor semántico de futuro, atenderemos igualmente a dos indicadores que se correlacionan con el grado de avance de un proceso de gramaticalización. Para ello, nos basaremos en la definición estándar de gramaticalización como aquel proceso por el cual una forma léxica pasa a adoptar un valor gramatical, y una forma gramatical adquiere un valor todavía más gramatical (Heine et al., 1991; Hopper y Traugott, 1993; Bybee et al., 1994; entre otros). Uno de estos indicadores, de carácter formal, corresponde a la posición de los clíticos respecto de las formas verbales que integran la perífrasis; el otro, de tipo semántico, alude al aumento del rasgo humano en los sujeto de las perífrasis (Bybee y Pagliuca, 1987; Bybee et al., 1994; Bybee, 2003). Los comentaremos brevemente a continuación.

\subsubsection{Posición del clítico}

Como es sabido (Fernández de Castro, 1999, Carrasco y García Fernández, 2006), los grupos verbales que forman perífrasis en español se distinguen de otras combinaciones no perifrásticas en que solo las primeras admi- 
ten la subida de clíticos, es decir, la adjunción de un pronombre átono a la forma finita, en lugar de la forma no finita (sea gerundio o infinitivo) que lo selecciona temáticamente ${ }^{6}$. Obsérvense los siguientes contrastes:

(4) a. Estoy leyéndolo

/ Lo estoy leyendo.

b. Voy a leerlo

/ Lo voy a leer.

c. Quiero leerlo

/ Lo voy a leer.

d. Tengo que leerlo

/ Lo tengo que leer.

(b) a. Temo decirlo

/ ??Lo temo decir.

b. Aspiro a solucionarlo

/ *Lo aspiro a solucionar.

c. Prefiero olvidarlo

/ ??Lo prefiero olvidar.

En los ejemplos de (4), se observa que el pronombre clítico puede aparecer bien junto a la forma no finita que lo selecciona argumentalmente, bien junto al verbo conjugado. Esta libertad posicional está severamente más restringida en el caso de los grupos verbales de (5). Sea que el resultado se perciba como inaceptable o plenamente gramatical, la diferencia con los grupos de (4) es clara. Este contraste se torna natural si se asume que, en el caso de una perífrasis, ambas formas verbales corresponden a un único predicado que proyecta una misma red argumental. La subida del clítico ilustra, en este caso, un reflejo formal del reanálisis morfosintáctico llevado a cabo, en el que tratamos la combinación de auxiliar y auxiliado como una misma pieza léxica sometida a flexión verbal, caso en el que la posición proclítica es la más aceptable ${ }^{7}$. Así, si bien se suele asumir que las perífrasis ofrecen una cierta libertad en la posición del clítico, veremos aquí que el mayor grado de avance de un proceso de gramaticalización se correlaciona con un aumento en la frecuencia del uso proclítico. Es decir, si bien las formas voy a pergarle y le voy a pegar son ambas aceptables, veremos que la consolidación de ir $a+$ inf como exponente gramaticalizado de futuro va de la mano con una marcada preferencia por el uso proclítico o preverbal.

6 Este criterio no es aplicable a las perífrasis de participio, toda vez que el participio no aloja formas clíticas en español (v.gr. lo he leído / *he leídolo). Así mismo, conviene destacar que la posición del clítico tampoco es libre en el caso de la perífrasis haber que + inf, por restricciones independientes del verbo haber en las que no podemos profundizar aquí. Así, no es gramatical la secuencia *lo hay que hacer.

7 El uso enclítico del pronombre en los verbos finitos con flexión temporal (v.gr. enfadóse, pensólo) se percibe hoy como profundamente arcaico. Por ello, la disposición que sirve como modelo de un predicado simple es la proclítica (se enfadó, lo pensó). 


\subsubsection{Semántica del sujeto}

Otro rasgo comúnmente observado en las combinaciones verbales perifrásticas frente a los grupos que no lo son es la renuncia a las restricciones de selección del auxiliar, que se limita a filtrar la red argumental del verbo auxiliado en las funciones sintácticas de la oración (Heine, 1993; Fernández de Castro, 1999; Bosque y Gutiérrez-Rexach, 2009). Dos consecuencias gramaticales de ello son la admisibilidad de verbos auxiliados impersonales (6) y la conservación de las relaciones temáticas en la pasiva (7):

(6) a. "Entonces va a haber una vorágine que te agarra" (Corpus B, H, 42 años)

b. *Intentará \{haber problemas / llover\}.

(7) a. El fiscal va a perseguir a los delincuentes.

a. Los delincuentes van a ser perseguidos por el fiscal.

b. El fiscal intentará perseguir a los delincuentes.

b. \#Los delincuentes intentarán ser perseguidos por el fiscal.

Como podemos observar, ir a admite su combinación con haber y llover, ambos impersonales, mientras que intentar, que selecciona su propio sujeto, y al que asigna un papel temático agentivo, los rechaza. En el caso de (7a), vemos que el cambio diatético efectuado por la pasiva del infinitivo no se ve alterado por el auxiliar; así, el fiscal sigue siendo el agente de (7a'), aunque ya no ocupe la posición de sujeto. En tanto, si el verbo proyecta su propia red argumental, como sucede en ( $7 \mathrm{~b})$, impedirá que el sujeto de la oración principal reciba el papel temático que sería esperable como sujeto del infinitivo en voz pasiva; así, (7b') altera el significado de (7b), indicando que los delincuentes poseen la intención de ser perseguidos por el fiscal.

Con verbos auxiliares altamente gramaticalizados, como ir en la construcción revisada, tales criterios se cumplen, según muestran los ejemplos arriba citados. Sin embargo, se ha observado que el curso de gramaticalización del futuro exhibe, a este respecto, una peculiaridad. De acuerdo con Bybee et al. (1994), los candidatos a expresar futuro pasan necesariamente, antes de indicar predicción (es decir, futuro), y una vez que se debilita el valor conceptual de la fuente léxica de la que proceden, por una etapa intermedia de intención (véase figura 1 supra \$1.1). Esto significa que, si bien en las etapas finales del proceso un verbo auxiliar que expresa futuro renunciará a toda restricción seleccional y dejará la proyección de la red argumental al verbo no finito, esperamos que, en 
las etapas intermedias, se produzca un aumento de la preferencia por sujetos capaces de volición e intención, es decir, sujetos humanos. En el caso de la gramaticalización de shall y will en inglés este parece haber sido justamente el caso, al verificarse, en textos del siglo XIV, un aumento en la frecuencia de sujetos de primera persona, contexto en el que el valor de intención se ve favorecido (Bybee et al., 1994; cf. Traugott, 1972, p.198 $)^{8}$. Dicho patrón general puede esquematizarse del modo siguiente ${ }^{9}$ :

Figura 4

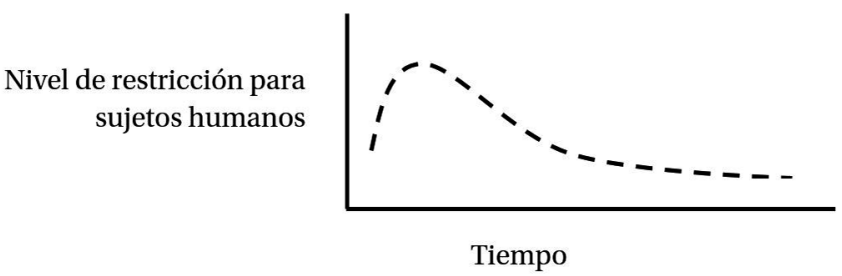

\subsection{Metodología y selección del corpus}

Como se ha adelantado en apartados anteriores, en este estudio se analizaron dos corpus orales del español de Chile. El primero de ellos (corpus A) corresponde a una selección de textos de Rabanales y Contreras (1979) integrada por 14 entrevistas realizadas en 1970, mientras que el segundo (corpus B) posee la misma cantidad de entrevistas, realizadas en 2009 para un estudio cualitativo sobre la estructura social chilena (Mayol Azócar y Azócar, 2013). En ambos casos, se trata de preguntas abiertas. La cantidad de palabras de cada corpus, así como su distribución en las categorías de género y nivel de instrucción se sintetizan en la tabla siguiente ${ }^{10}$ :

$8 \mathrm{Al}$ respecto, señala Bybee (2003, p.251): "Una etapa intermedia de intención, especialmente con los sujetos de primera persona, queda bien documentada en todas las formas de las que deriva el futuro". 9 Nótese que esta curva en el cambio de restricciones seleccionales no tiene por qué extenderse a otros procesos de gramaticalización de formas perifrásticas que expresan tiempo. Así, no es de esperar que, por ejemplo, la gramaticalización de una construcción resultativa con tener hacia un valor de perfecto (y eventualmente aoristo) haya de pasar por una etapa intermedia de aumento de sujetos humanos, toda vez que el proceso conceptual involucrado (inferencia desde el estado resultante al evento anterior) no exige necesariamente un rasgo semántico específico en el sujeto gramatical.

10 En el presente estudio se procuró, siempre que los datos disponibles así lo permitieran, que las variables de género y nivel de formación fuesen equiparables para ambos grupos. No hemos alcanzado una simetría perfecta entre las dos muestras debido al carácter diverso de las investigaciones de las que ellas proceden. El total de palabras de cada corpus se ha obtenido restando el número de palabras correspondientes al discurso de los entrevistadores. 
Tabla 1.

Conformación de los corpus seleccionados.

\begin{tabular}{|c|c|c|c|c|c|c|}
\hline & $\begin{array}{c}\text { No. de } \\
\text { palabras }\end{array}$ & Hombres & Mujeres & $\begin{array}{c}\text { Nivel de } \\
\text { instrucción } \\
\text { alto } \\
\text { (universitario } \\
\text { completo) }\end{array}$ & $\begin{array}{c}\text { Nivel de } \\
\text { instrucción } \\
\text { medio } \\
\text { (escolaridad } \\
\text { completa) }\end{array}$ & $\begin{array}{c}\text { Nivel de } \\
\text { instrucción } \\
\text { bajo } \\
\text { (escolaridad } \\
\text { incompleta) }\end{array}$ \\
\hline $\begin{array}{c}\text { Corpus A } \\
\text { (1970) }\end{array}$ & 53.600 & 7 & 7 & 14 & 0 & 0 \\
\hline $\begin{array}{c}\text { Corpus B } \\
\text { (2009) }\end{array}$ & 132.372 & 7 & 7 & 10 & 3 & 1 \\
\hline
\end{tabular}

A efectos de la medición de la frecuencia de las formas estudiadas, se procedió a contabilizar el total de formas a las que podría atribuirse un valor primario o inferencial de futuro, y que corresponden a las formas arriba descritas: futuro sintético en -ré, ir a + inf, tener que + inf, querer + inf y haber que + inf $^{11}$. El total de apariciones de estas formas integra, para cada corpus, lo que hemos denominado conjunto de referencia de futuro. La frecuencia de uso de una cierta construcción corresponde, así, a la frecuencia relativa de dicha forma respecto del conjunto de referencia de futuro de su propio corpus. El total de ocurrencias para ambos conjuntos es el siguiente:

Tabla 2.

Total de conjuntos de referencia de futuros.

\begin{tabular}{|l|c|}
\hline & Conjunto de referencia de futuro \\
\hline Corpus A (1970) & $\mathbf{2 4 0}$ (sobre 53.600 palabras) \\
\hline Corpus B (2009) & $\mathbf{9 7 3}$ (sobre 132.372 palabras) \\
\hline
\end{tabular}

La representatividad de cada forma en su conjunto de referencia de futuro se complementa con la medición de los indicadores de gramaticalización arriba expuestos: posición del clítico y semántica del sujeto.

En términos más específicos, los resultados presentados en la sección siguiente corresponden, además de a ocurrencias del futuro sintético, a formas perifrásticas en las que el auxiliar está conjugado en presente de indicativo, en cualquiera de sus personas gramaticales ( $\{$ voy/vas $/ v a /$ vamos/van\} a cantar). Esta restricción obedece, en lo fundamental, al in-

11 Esta última forma corresponde, de acuerdo a Fernández de Castro (1999), a una perífrasis impersonal, de modo que difícilmente pasaría a sustituir a la forma canónica de futuro. Sin embargo, hemos decidido considerarla por dos motivos: el primero es que posee una frecuencia relativamente alta; el segundo, que al expresar modalidad deóntica, permite evaluar mejor el aumento en la frecuencia de uso de tener que + inf, cuyo valor principal es igualmente deóntico. Volveremos sobre este punto en \$2.5. 
tento de delimitar un conjunto de expresiones que fuesen mínimamente equiparables. Aunque, como hemos argumentado más arriba, las formas tener que + inf, querer + inf y haber que + inf no denotan la localización del punto de referencia con posterioridad al momento de habla (ya que dicha inferencia es cancelable), es en el uso de presente de indicativo que este valor secundario puede emerger con mayor claridad y sustituir potencialmente bien a la forma sintética en -ré, bien a la forma analítica $i r$ $a+$ inf. Así, todas las formas pueden conjugarse en otros tiempos verbales (v.gr. tuvo que cantar, querrá cantar, había que cantar, iba a cantar), pero los valores temporales que en tales casos pueden emerger corresponden a otros tiempos del paradigma verbal (así sucede, por ejemplo, con la forma en imperfecto iba a cantar, que ha ido sustituyendo a cantaría en su valor indicativo de pospretérito ${ }^{12}$ ). Asimismo, la flexión del auxiliar en modo subjuntivo trastorna aún más la sustituibilidad por la forma sintética (v.gr. Cuando \{haya que / tengas que / quieras / vayas a\} cantar, avisame, ver supra $)^{13}$.

Finalmente, y siguiendo el objetivo de procurar una equiparación funcional de las formas escogidas, se han descartado ocurrencias de tener $q u e+$ inf que tuviesen un valor modal epistémico (v.gr. Tiene que estar ahora en su casa, ino?), toda vez que, en este contexto, la inferencia de futuro desaparece, tal como sucede con el uso epistémico del futuro sintético (v.gr. Estará ahora en su casa, ¿no?).

En suma, esperamos delinear una estrategia de seguimiento de un proceso de gramaticalización en la que el avance de una cierta forma se correlaciona, por un lado, con valores positivos en los diagnósticos de gramaticalización y, por otro, con fenómenos de reacomodo en el resto de las construcciones del conjunto de referencia de futuro. Somos conscientes, con todo, de que el tramo temporal que abarca la muestra (39

\footnotetext{
12 Se suele atribuir a la forma iba a + inf un valor de conato (v.gr. Lo descubrieron justo cuando iba a cometer el crimen $\rightarrow$ 'no se cometió el crimen', cf. Fernández de Castro, 1999, p.207) que, por el momento, la distanciaría del valor indicativo del condicional simple. Sin embargo, es posible encontrar casos donde la perífrasis en imperfecto posee un valor genuino de pospretérito, sin que se dé la presuposición de no ocurrencia del evento. Un ejemplo puede ser el siguiente: "Ness decidió que, cuando tuviera la oportunidad, Majidah iba a enterarse" (books.google.cl, Elizabeth George, Tres hermanos, recuperado: 10.10.16), donde la forma en cursiva es plenamente sustituible por se enteraría. Con todo, la gramaticalización de esta forma queda para futuras investigaciones.

13 Como hemos mencionado, la agramaticalidad del futuro de indicativo en cláusulas adverbiales introducidas por cuando es una restricción bastante clara en español; no obstante, resulta interesante considerar cómo, en este y otros contextos inductores de subjuntivo, se puede recuperar un tiempo largamente perdido del paradigma español: el futuro de subjuntivo, que en la actualidad ha sido sustituido, exceptuando el discurso jurídico, bien por el presente de subjuntivo (Cuando te cases...), bien por el imperfecto de subjuntivo (Si se casara...).
} 
años) es escaso con respecto al periodo que un proceso de esta naturaleza cubriría efectivamente, por lo que, de resultar informativos los datos aquí presentados, su validez como evidencia de las propuestas teóricas arriba expuestas debería considerar un tramo temporal más amplio. En nuestro caso, dicho objetivo mayor se ve limitado por la escasez de datos comparables en cortes sincrónicos previos, aunque estimamos que una profundidad histórica mayor puede alcanzarse si se consideran otros géneros discursivos, tomando en cuenta la estrategia de análisis aquí seguida.

\subsection{Resultados de los corpus analizados}

\subsubsection{Corpus A (1970)}

En la siguiente tabla se presenta la frecuencia relativa de las formas integrantes del conjunto de referencia de futuro del corpus A.

Tabla 3.

Frecuencia de formas en corpus A.

\begin{tabular}{|c|c|c|c|c|c|}
\hline $\begin{array}{c}\text { Conjunto de } \\
\text { referencia } \\
\text { de futuro } \\
(1970)\end{array}$ & $\begin{array}{c}\text { Futuro } \\
\text { sintético }\end{array}$ & $\begin{array}{c}\text { Ir a + } \\
\text { infinitivo }\end{array}$ & $\begin{array}{c}\text { Querer + } \\
\text { infinitivo }\end{array}$ & $\begin{array}{c}\text { Tener que + } \\
\text { infinitivo }\end{array}$ & $\begin{array}{c}\text { Haber que + } \\
\text { infinitivo }\end{array}$ \\
\hline 240 & 18 & 122 & 30 & 55 & 15 \\
\hline $100 \%$ & $7,5 \%$ & $50,8 \%$ & $12,5 \%$ & $22,9 \%$ & $6,2 \%$ \\
\hline
\end{tabular}

Según puede observarse, la forma predominante, con un 50,8\% de ocurrencias, es la perífrasis ir $a+\mathrm{inf}$, seguida de tener que + inf, con un 22,9\%. La forma sintética, en tanto, representa un 7,5\% del conjunto. A este respecto, es preciso señalar que dicho porcentaje se restringe a las ocurrencias de -ré con valor temporal. La forma posee en total $42 \mathrm{ocu}-$ rrencias, de las cuales 24 , es decir, un $57 \%$, corresponden a usos modales epistémicos en los que se pierde toda referencia al futuro ${ }^{14}$. Esta distribución se aprecia en la siguiente tabla:

14 No podemos desarrollar aquí en detalle las restricciones del uso modal epistémico del morfema -ré, que está restringido a predicados estativos (como ilustra el ejemplo citado en (8)) y se ve bloqueado con predicados eventivos (Juan cantará una cumbia, con lectura temporal excluyente). Para más detalles, véase Jaque (2016) y las referencias allí citadas. 
Tabla 4.

Distribución de valores temporales y modales de -ré en corpus A.

\begin{tabular}{|c|c|c|}
\hline Formas de futuro sintético & Valor temporal & Valor modal epistémico \\
\hline Total: 42 & 18 & 24 \\
\hline $100 \%$ & $42,8 \%$ & $57,1 \%$ \\
\hline
\end{tabular}

Un ejemplo de uso epistémico del futuro se ofrece a continuación:

(8) La religión... no sé si seré cristiano o no. (Corpus A, Hombre, 25 años)

Esta tendencia, según tendremos ocasión de comprobar, se afirma en los resultados del corpus $\mathrm{B}$, indicando la consolidación que, en la oralidad, posee el uso epistémico de -ré y su decadencia como marcador de futuro.

Respecto de la semántica del sujeto, se presentan los resultados en la tabla siguiente:

Tabla 5 .

Semántica del sujeto en formas del corpus A.

\begin{tabular}{|l|l|c|c|c|c|c|}
\hline & Futuro & Ir $a+$ inf & $\begin{array}{c}\text { Querer + } \\
\text { inf }\end{array}$ & $\begin{array}{c}\text { Tener que } \\
+ \text { inf }\end{array}$ & $\begin{array}{c}\text { Haber que } \\
+ \text { inf }\end{array}$ \\
\hline $\begin{array}{l}\text { Semántica } \\
\text { del sujeto }\end{array}$ & $\begin{array}{l}\text { Suj } \\
\text { humano }\end{array}$ & $12(66,6 \%)$ & $93(76,2 \%)$ & $29(96,6 \%)$ & $40(72,7 \%)$ & - \\
\cline { 2 - 7 } & $\begin{array}{l}\text { Suj no } \\
\text { humano }\end{array}$ & $6(33,3 \%)$ & $29(23,7 \%)$ & $1(3,3 \%)$ & $15(27,2 \%)$ & $15(100 \%)$ \\
\hline
\end{tabular}

Este criterio no es aplicable a la forma haber que + inf, toda vez que se trata de una forma impersonal. Con respecto al resto de las perífrasis, se observa un predominio del rasgo humano. No obstante, por lo pronto, solo en el caso de querer + inf puede destacarse una preferencia marcada por sujetos volitivos. En el caso del resto, conviene observar comparativamente los resultados, una vez que presentemos los datos del corpus B, para apreciar posibles tendencias.

A continuación, presentamos la distribución de los clíticos para aquellas construcciones que los presentan. Dicho criterio no es, nuevamente, relevante en el caso de haber que + inf, ni tampoco en el de -ré, que se excluye en la presente tabla: 
Tabla 6.

Posición del clítico en formas del corpus A.

\begin{tabular}{|l|l|c|c|c|c|}
\hline \multicolumn{2}{|l|}{} & Ir $a+$ inf & Querer + inf & $\begin{array}{c}\text { Tener que } \\
+ \text { inf }\end{array}$ & $\begin{array}{c}\text { Haber que } \\
+ \text { inf }\end{array}$ \\
\hline $\begin{array}{l}\text { Posición del } \\
\text { clítico }\end{array}$ & $\begin{array}{l}\text { Antepuesto } \\
\text { al auxiliar }\end{array}$ & $44(100 \%)$ & $5(55,6 \%)$ & $5(25 \%)$ & - \\
\cline { 2 - 6 } & $\begin{array}{l}\text { Pospuesto al } \\
\text { infinitivo }\end{array}$ & o (o\%) & $4(44,4 \%)$ & $15(75 \%)$ & $4(100 \%)$ \\
\hline \multicolumn{2}{|l|}{ Total clíticos: 77} & 44 & 9 & 20 & 4 \\
\hline
\end{tabular}

Tal como anunciábamos, se aprecia una marcada preferencia por el uso proclítico en ir $a+$ inf. En el caso de las perífrasis restantes, la distribución es más pareja. Tener que + inf exhibe una preferencia por la posición enclítica, mientras que, para el caso de querer + inf, el establecimiento de una tendencia resulta más conflictivo, dado el número más reducido de casos totales.

\subsubsection{Corpus B (2009)}

Veamos, a continuación, los resultados del corpus B (2009). En la tabla siguiente, se observa la distribución general de las formas que integran el correspondiente conjunto de referencia de futuro:

Tabla 7.

Frecuencia de formas de futuro en corpus B.

\begin{tabular}{|c|c|c|c|c|c|}
\hline $\begin{array}{c}\text { Conjunto de } \\
\text { referencia de } \\
\text { futuro (2009) }\end{array}$ & $\begin{array}{c}\text { Futuro } \\
\text { sintético }\end{array}$ & $\begin{array}{c}\text { Ir } a+ \\
\text { infinitivo }\end{array}$ & $\begin{array}{c}\text { Querer }+ \\
\text { infinitivo }\end{array}$ & $\begin{array}{c}\text { Tener que + } \\
\text { infinitivo }\end{array}$ & $\begin{array}{c}\text { Haber que } \\
+ \text { infinitivo }\end{array}$ \\
\hline 973 & 5 & 523 & 98 & 288 & 59 \\
\hline $100 \%$ & $0,5 \%$ & $53,7 \%$ & $10 \%$ & $29,5 \%$ & $6 \%$ \\
\hline
\end{tabular}

Puede apreciarse la escasa representatividad que posee la forma sintética en -ré, con menos de un $1 \%$ de las ocurrencias. En cambio, se consolida la perífrasis ir $a+$ inf como la expresión predominante del futuro en español. Con respecto a las dos formas restantes, cabe destacar el incremento en la frecuencia de tener que + inf, que pasa de un $22,9 \%$ a casi un $30 \%$ de las ocurrencias.

Con respecto a la forma sintética, conviene una vez más tomar en cuenta el contraste con los usos epistémicos, que en este caso suman 28 ocurrencias, correspondientes al 84,8\% de las 33 que suman los usos de futuro sintético en el corpus: 
Tabla 8.

Distribución de valores temporales y modales de -ré en corpus B.

\begin{tabular}{|c|c|c|}
\hline Formas de futuro sintético & Valor temporal & Valor modal epistémico \\
\hline Total: 33 & 5 & 28 \\
\hline $100 \%$ & $15,2 \%$ & $84,8 \%$ \\
\hline
\end{tabular}

Esto viene a confirmar,pues, la consolidación del morfema -ré como exponente de modalidad epistémica frente a la práctica desaparición de su empleo como marca de tiempo futuro.

A continuación, se presentan los valores relativos a la semántica de los sujetos correspondientes a cada forma, que tendremos ocasión de comentar con más detalles en el subapartado siguiente:

Tabla 9.

Semántica del sujeto en formas del corpus B.

\begin{tabular}{|c|c|c|c|c|c|c|}
\hline & & $\begin{array}{c}\text { Futuro } \\
\text { sintético }\end{array}$ & $\begin{array}{c}\text { Ir } a+ \\
\text { inf }\end{array}$ & $\begin{array}{c}\text { Querer+ } \\
\text { inf }\end{array}$ & $\begin{array}{l}\text { Tener que } \\
+ \text { inf }\end{array}$ & $\begin{array}{l}\text { Haber que } \\
+ \text { inf }\end{array}$ \\
\hline \multirow[t]{2}{*}{$\begin{array}{l}\text { Semántica } \\
\text { del sujeto }\end{array}$} & $\begin{array}{l}\text { Suj } \\
\text { humano }\end{array}$ & $\begin{array}{c}1 \\
(20 \%)\end{array}$ & $\begin{array}{c}423 \\
(80,8 \%)\end{array}$ & $\begin{array}{c}94 \\
(95,8 \%)\end{array}$ & $\begin{array}{c}260 \\
(90,3 \%)\end{array}$ & - \\
\hline & \begin{tabular}{|l} 
Suj no \\
humano
\end{tabular} & $\begin{array}{c}4 \\
(80 \%)\end{array}$ & $\begin{array}{c}100 \\
(19,2 \%)\end{array}$ & $\begin{array}{c}4 \\
(4,2 \%)\end{array}$ & $\begin{array}{c}28 \\
(9,7 \%)\end{array}$ & $\begin{array}{c}59 \\
(100 \%)\end{array}$ \\
\hline
\end{tabular}

Finalmente, la tabla 10 muestra los datos referentes a la posición del clítico, en la que se confirma la correlación entre el predominio de la perífrasis ir $a+$ inf y la preferencia por la posición antepuesta al auxiliar.

Tabla 10.

Posición del clítico en formas del corpus B.

\begin{tabular}{|l|l|c|c|c|c|}
\hline \multicolumn{2}{|l|}{} & Ir a + inf & Querer+inf & $\begin{array}{c}\text { Tener que } \\
+ \text { inf }\end{array}$ & $\begin{array}{c}\text { Haber que } \\
+ \text { inf }\end{array}$ \\
\hline $\begin{array}{l}\text { Posición del } \\
\text { clítico }\end{array}$ & $\begin{array}{l}\text { Antepuesto } \\
\text { al auxiliar }\end{array}$ & $190(93,5 \%)$ & $17(60,8 \%)$ & $32(37,3 \%)$ & - \\
\cline { 2 - 6 } & $\begin{array}{l}\text { Pospuesto al } \\
\text { infinitivo }\end{array}$ & $13(6,5 \%)$ & $11(39,2 \%)$ & $54(62,7 \%)$ & $18(100 \%)$ \\
\hline \multicolumn{2}{|l|}{ Total clíticos: 335} & 203 & 28 & 86 & 18 \\
\hline
\end{tabular}

\subsection{Discusión y comparación}

En el presente apartado comentaremos los puntos de contraste que muestran los datos presentados en el apartado anterior y que, a la luz de los conceptos introducidos más arriba, pueden ilustrar un mecanismo de 
gramaticalización en curso en el que los distintos exponentes no evolucionan de forma aislada. En el gráfico 1 se muestra la distribución de las formas seleccionadas en ambos momentos sincrónicos:

\section{Gráfico 1.}

Comparación de la frecuencia de las formas en ambos corpus.

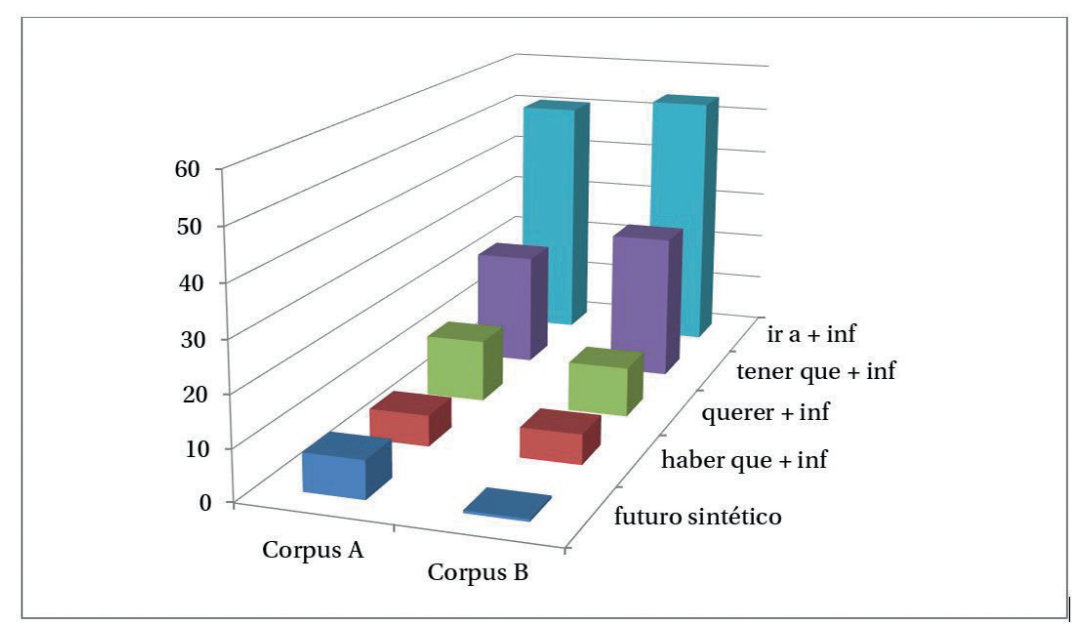

Es interesante notar que, junto a la posición relativamente estable que poseen las perífrasis ir a + inf, querer + inf y haber que + inf, existen alteraciones importantes en la representatividad de la forma sintética en -ré y en la perífrasis tener que + inf. Así, mientras que la primera parece desaparecer por completo del registro oral, la segunda sufre, en cambio, un aumento. Así, tener que + inf se consolida como la segunda perífrasis en el conjunto de referencia de futuro. La pregunta que cabe hacerse es, por consiguiente, si este aumento en la frecuencia se correlaciona con valores positivos en otros indicadores de gramaticalización, que puedan sugerir que esta perífrasis constituye un "buen candidato" para una eventual formación de un exponente de tiempo simbólico. No sería aventurado pensar que este sea el caso, toda vez que la perífrasis ir $a+$ inf parece haber desplazado definitivamente al antiguo exponente de tiempo simbólico, perdiendo, en el proceso, las características de tiempo fenomenológico (cercanía con el presente dada por el aspecto inminencial) que la hicieran en un principio una buena alternativa a -ré. En primer lugar, debemos descartar que el aumento de tener que + inf se deba únicamente a un aumento generalizado de las formas que expresan modalidad deóntica. 
Si se observa el gráfico 1, se aprecia que la frecuencia de haber que + inf, que expresa igualmente obligación, es exactamente la misma en ambos corpus (6\%). De este modo, el aumento de la frecuencia de tener que + inf podría interpretarse como un proceso de cambio incipiente que afecte de modo particular a esta perífrasis.

El siguiente gráfico, en el que se muestra comparativamente la posición del clítico, apunta en esta dirección. Se ilustran aquí las posiciones de los clíticos para las dos perífrasis que, por motivos históricos, podemos considerar como buenos candidatos para transformarse en exponentes de futuro.

Gráfico 2.

Posición del clítico en tener que + inf y querer + inf en ambos corpus.

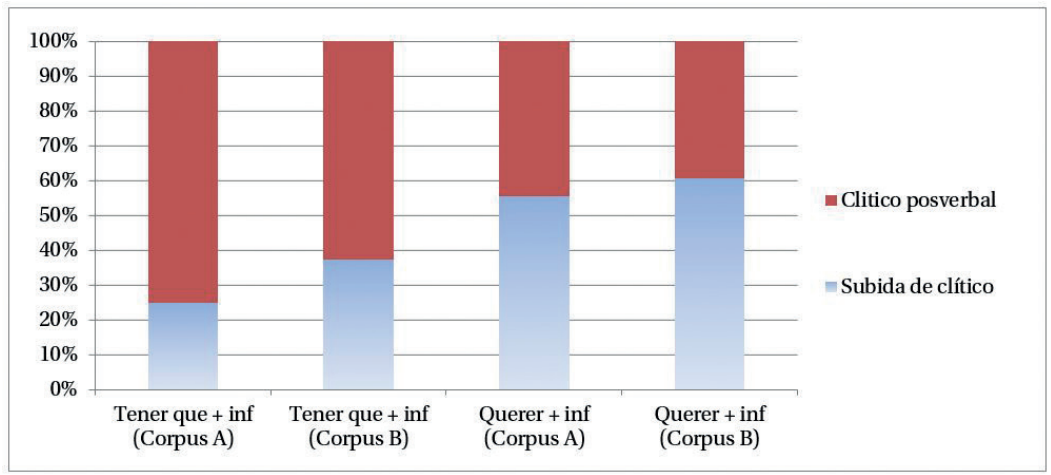

Como puede observarse, la tendencia es, en ambos casos, a incrementar la preferencia por la posición preverbal (subida de clítico), cuestión que resulta más llamativa en el caso de tener que + inf, que pasa desde un $25 \%$ a un $37 \%$.

Algo similar sucede si consideramos el indicador de la semántica del sujeto. Nuevamente, querer + inf posee una alta selección de sujetos humanos que se mantiene en ambos corpus. Sin embargo, esta selección semántica es propia del verbo aun cuando no opere en un conjunto perifrástico y se emplee en cambio como verbo pleno (v.gr. quiero una bicicleta). De este modo, el efecto que el proceso de gramaticalización tenga respecto del alza de restricciones seleccionales se vuelve en este punto opaco ${ }^{15}$.

15 En cualquier caso puede apreciarse un ocasional relajamiento en las restricciones seleccionales 
Gráfico 3 .

Semántica del sujeto en tener que + inf y querer + inf en ambos corpus.

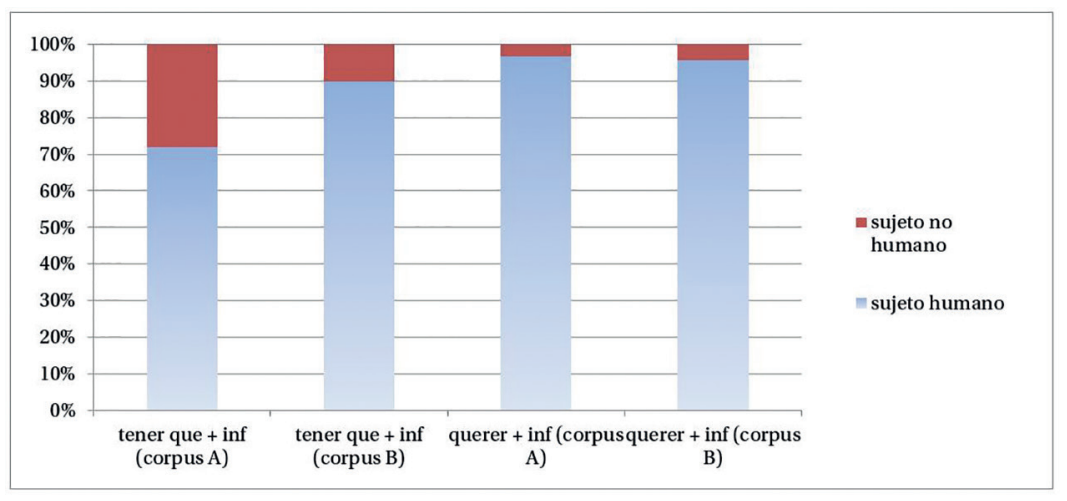

Es distinto el caso de tener que + inf. En principio, no ofrece tales restricciones seleccionales ni como verbo independiente (v.gr. La mesa tiene cuatro patas), ni como operador de modalidad, sea deóntica (v.gr. Tiene que llover mañana) o epistémica (v.gr. Tiene que estar lloviendo afuera, porque se oye mucho ruido). Por lo tanto, en este caso sí resulta significativo que se produzca un aumento en la frecuencia de sujetos humanos, que pasan de representar el $72 \%$ a un $90 \%$.

De este modo, nos vemos ante tres hechos que parecen indicar cierta preferencia por la perífrasis tener que + inf como un buen candidato para reiniciar el proceso de compensación entre tiempo simbólico y tiempo fenomenológico que, de acuerdo con nuestras asunciones, debiese secundar a la culminación del proceso de gramaticalización de $i r$ $a+$ inf. El primero y más evidente es la frecuencia de uso: corresponde a la segunda perífrasis más empleada, en ambos corpus, tendencia que aumenta para 2009. Por otra parte, vemos que este aumento de frecuencia va acompañado de valores positivos en dos indicadores de gramaticalización: la subida del clítico (o posición proclítica) y el aumento de la selección de sujetos humanos.

En ambos indicadores, los valores de querer + inf son igualmente positivos, pero sufren menos incrementos a través del tiempo, cuestión que 
se correlaciona con que dicha perífrasis sea menos frecuente en ambos corpus.

\section{Conclusiones}

En este trabajo hemos abordado el proceso de gramaticalización de las formas de futuro en dos momentos del español contemporáneo de Chile. Hemos partido del supuesto teórico de que la formación de perífrasis de futuro no se explica de modo suficiente ni sobre la base de una concepción pragmático-discursiva de la gramaticalización (Bybee et al., 1994), ni sobre el empleo de metáforas conceptuales (Melis et al., 2003, entre otros). En cambio, hemos propuesto que los cambios en el sistema de expresión de la temporalidad están motivados por la compensación entre dos formas de cognición temporal: tiempo fenomenológico, es decir, la experiencia directa y centrada en el presente; y tiempo simbólico, esto es, la organización secuencial deíctica de intervalos y eventos con independencia de la experiencia del hablante. Esta segunda forma de percepción temporal es derivada de la primera. Así, decimos, siguiendo a Fleischman (1982), que los exponentes de tiempo fenomenológico, en los que priman contenidos modales y aspectuales, derivan en exponentes de tiempo simbólico, en un proceso en el que las distintas formas establecen una compensación semántica (y en última instancia, cognitiva).

Al ponderar estas ideas para los datos seleccionados del español de Chile, hemos podido observar los siguientes hechos:

La forma ir $a+$ inf se consolida, en la oralidad, como el exponente de tiempo simbólico de futuro por excelencia, cuestión que se refleja tanto en su alta frecuencia de uso como en la preferencia por la subida del clítico. En términos semánticos, la localización futura del evento denotado por el verbo no finito no es cancelable.

El avance de ir $a+$ inf parece haber desplazado definitivamente a -ré, que, en el español sincrónico oral, se especializa para la expresión de la modalidad epistémica.

La finalización del proceso de gramaticalización tendría, como especulamos a partir de los datos disponibles, un efecto en la puesta en marcha de la gramaticalización de otras formas perifrásticas disponibles que pueden ser "buenos candidatos" para expresar futuro. De las construcciones que hemos revisado, se evidencia una preferencia por la perífrasis tener que + inf, cuestión que se refleja en su alta frecuencia de uso, así 
como en el aumento tanto de la posición preverbal del clítico como en el incremento de las restricciones seleccionales para sujetos humanos. De todas formas, la inferencia de futuro es en este caso cancelable, prueba de que aún no pasa a recategorizarse como el significado primario de la forma. Si nuestra aproximación es acertada, prevemos que este cambio podría tener lugar a medida que se agudice la desaparición de la forma sintética (en otros registros, por ejemplo) y se debiliten aún más los valores aspectuales asociados a la opción perifrástica en $i r$.

En síntesis, creemos que el examen de los corpus presta fuerza a la idea de que, al menos en el dominio de la expresión temporal, la gramaticalización de las distintas unidades léxicas no se da de forma aislada, sino que opera como un conjunto armónico guiado, en última instancia, por motivaciones cognitivas relacionadas con nuestra percepción temporal. Con todo, según hemos expresado en 2.3, la indagación aquí efectuada busca ofrecer una estrategia de análisis, más que una confirmación empírica. Esta debiese tomar en cuenta corpus comparables pertenecientes a cortes sincrónicos que abarquen una ventana de tiempo mayor.

\section{Bibliografía}

Bentivoglio, P. \& Sedano, M. (2015). Morphosyntactic Variation in Spanish-Speaking Latin America. En M. Díaz-Campos (ed.) The Handbook of Hispanic Sociolinguistics (pp. 168-186). Sussex: Wiley Blackwell.

Bosque, I. \& Gutiérrez-Rexach, J. (2009). Fundamentos de sintaxis formal. Madrid: Akal.

Bybee, J. (1998). Cognitive Processes in Grammaticalization. En M. Tomasello (ed.) The New Psychology of Language. Cognitive and Functional Approaches to Language Structure, vol. II (pp. 145-167). Londres: Lawrence Erlbaum Associates.

Bybee, J. (2003). Los mecanismos de cambio como universales lingüísticos. En R. Mairal \& J. Gil (eds.) En torno a los universales lingüísticos (pp. 245-263). Madrid: Akal.

Bybee, J. \& Pagliuca, W. (1987). The evolution of future meaning. En A. Giacalone, O. Garruba, y G. Bernini (eds.) Papers from the VIIth International Conference on Historical Linguistics (pp. 109-122). Amsterdam: John Benjamins. 
Bybee, J., Pagliuca, W. \& Perkins, R. (1991). Back to the future. En E. Traugott \& B. Heine (eds.) Aproaches to grammaticalization, Vol. II (pp. 17-58). Amsterdam: John Benjamins.

Bybee, J., Perkins, R. \& Pagliuca, W. (1994). The evolution of Grammar. Tense, Aspect and Modality in the Languages of the World. Chicago y Londres: The University Chicago Press.

Carrasco, A. \& García Fernández, L. (2006). Diccionario de perífrasis verbales. Madrid: Gredos.

Cartagena, N. (1995-1996). La inestabilidad del paradigma verbal de futuro, ¿hispanoamericanismo, hispanismo o universal lingüístico? Boletín de Filología, XXXV, 79-100.

Comrie, B. (1985). Tense. Cambridge: CUP.

Cuenca, M. J. \& Hilferty, J. (2013). Introducción a la lingüística cognitiva. Barcelona: Ariel.

Escandell, M. (2010). Futuro y evidencialidad. Anuario de Lingüística Hispánica, 26, 9-34.

Evans, V. (2005). The Structure of Time. Language, meaning and temporal cognition. Amsterdam/Philadelphia: John Benjamins.

Fernández de Castro, F. (1999). Las perifrasis verbales en el español actual. Madrid: Gredos.

Fleischman, S. (1982). The future in thought and language. Diachronic evidence from Romance. Londres: Cambridge University Press.

Grady, J. (1997). Foundations of meaning: Primary metaphors and primary scenes (Tesis doctoral). U.C. Berkeley.

Heine, B. (1993). Auxiliaries. Cognitive Forces and Grammaticalization. Nueva York, Oxford: Oxford University Press.

Heine, B, Claudi, U. \& Hünnemeyer, F. (1991). Grammaticalization: A conceptual framework. Chicago: University of Chicago Press.

Hopper, P. \& Traugott, E. Grammaticalization. Cambridge: Cambridge University Press.

Husserl, E. (1905 [2002]). Lecciones de fenomenología de la conciencia interna del tiempo. Traducción, introducción y notas de Agustín Serrano de Haro. Madrid: Trotta.

Jaque, M. (2012). Tiempo y lenguaje. Restricciones cognitivo-fenomenológicas en la evolución de las formas de futuro (Tesis de magíster). Universidad de Chile. 
Jaque, M. (2016). Relaciones entre aspecto y modalidad epistémica: algunas consecuencias de las restricciones temporales sobre la evaluación de predicados. Onomázein, 33, 130-155.

Klein, W. (1994). Time in language. London \& Nueva York: Routledge.

Lakoff, G. \& Johnson, M. (1980). Metaphors we live by. Chicago: University Press.

Lastra, Y. \& Martín-Butragueño, P. (2010). Futuro perifrástico y futuro morfológico en el corpus sociolingüístico de la ciudad de México. Oralia, 13, 145-171.

Mayol, A., Azócar, C. \& Azócar, C. (2013). El Chile profundo. Modelos culturales de la desigualdad y sus resistencias. Santiago: Liberalia.

Melis, C. (2006). Verbos de movimiento. La formación de los futuros perifrásticos. En C. Company (dir.) Sintaxis histórica de la lengua española, vol. 2 (pp. 873-968). México: Fondo de Cultura Económica.

Melis, C., Flores, M. \& Bogard, S. (2003). La Historia del Español. Propuesta de un tercer periodo evolutivo. Nueva Revista de Filología Hispánia, Tomo LI, no. 1, 32-68.

Orozco, R. (2007). Social constraints on the expression of futurity in spanish-speaking urban communities. En J. Holmquist \& L. Sayahi (eds.) Selected proceedings of the third workshop on spanish linguistics (pp. 103-112). Somerville, MA: Cascadilla Proceedings Project.

Pöppel, E. (1994). Temporal mechanisms in perception. En O. Sporns \& G. Tononi (eds.) Selectionism and the brain: Internaional review of neurobiology, vol. 37 (pp. 185-201). San Diego, CA: Academic Press.

Rabanales, A. \& Contreras, L. (1979). El habla culta de Santiago de Chile. Materiales para su estudio. En Boletín de Filología, Anejo n. 2.

Sedano, M. (1994). El futuro morfológico y la expresión ir a + infinitivo en el español hablado de Venezuela. Verba, 21, 225-240.

Traugott, E. (1972). The History of English Syntax. Nueva York: Holt, Rinehart and Winston.

Varela, F. (1995). The Specious Present: A Neurophenomenology of Time Consciousness. En J. Petitot, F. Varela, B. Pacoud \& J. Roy (eds.), 
Naturalizing Phenomenology (pp. 266-314). Stanford: Standford University Press.

Varela, F., E. Thompson \& E. Rosch (1991). The embodied mind: Cognitive Science and human experience. Cambridge, MA: MIT Press.

Zahavi, D. (2003). Inner Time-Consciousness and Pre-reflective Selfawareness. En D. Welton (ed.) The new Husserl. A critical reader (pp. 157-180). Indianapolis: Indiana University Press. 